\title{
MASS-LOADING AND PARALLEL MAGNETIZED SHOCKS
}

\author{
G.P. Zank ${ }^{1}$, S. Oughton ${ }^{1}$, F.M. Neubauer ${ }^{2}$, and G.M. Webb ${ }^{3}$
}

Abstract. Recent observations at comets Giacobini-Zinner and Halley suggest that simple non-reacting gas dynamics or MHD is an inappropriate description for the bow shock. The thickness of the observed (sub)shock implies that mass-loading is an important dynamical process within the shock itself, thereby requiring that the RankineHugoniot conditions possess source terms. This leads to shocks with properties similar to those of combustion shocks. We consider parallel magnetized shocks subjected to massloading, describe some properties which distinguish them from classical MHD parallel shocks, and establish the existence of a new kind of MHD compound shock. These results will be of importance both to observations and numerical simulations of the comet-solar wind interaction.

\section{Introduction}

Photoionization of the extensive neutral coma surrounding a comet leads to mass-loading of the solar wind. Since the earliest investigations [Axford, 1964; Biermann et al., 1967 (BBS)], it was recognized that mass-loading would decelerate a supersonic flow, and, in particular, BBS showed that this deceleration continued until a critical mass-loading rate was achieved. Thereafter, for the flow to reach a final downstream state, it was necessary to insert a (sub)shock into the fluid flow. These theoretical results sparked an extended debate concerning the existence, location and strength of such a shock, with Wallis [1971] suggesting that either no shock existed and that the flow was completely smooth ("a bow wave") or that the shock was considerably weaker than that suggested by BBS. Recent detailed numerical simulations [e.g., Ogino et al., 1988] of the interaction between the solar wind and cometary atmospheres provide support for the existence of a weak bow shock. Furthermore the recent missions to comets Giacobini-Zinner (GZ) and Halley have shed considerable light on both the micro- and macrophysics of the cometary interaction with the solar wind [e.g., Ip \& Axford, 1989], and observations made during the Halley encounter provide clear evidence for the existence of a shock [e.g., Neubauer et al., 1986; Coates et al., 1987].

The slightly decelerated flow regime ahead of the shock appears to be reasonably consistent with the gross predictions of fluid dynamical models, but the observed structure of the embedded shock is very different from the simple

Bartol Research Institute, University of Delaware
2Institut für Geophysik und Meteorlogie, Universität Köln
${ }^{3}$ Department of Planetary Sciences, University of Arizona

Copyright 1991 by the American Geophysical Union.

Paper number 91 GL02242

0094-8534/91/91GL-02242\$03.00 gas dynamical subshock picture of a sharp, discontinuous transition. Instead, observations at GZ and Halley reveal that

1. the shock transitions tend to be very broad-e.g., the shock thickness on the inbound (outbound) Halley encounter was found to be $\sim 45000 \mathrm{~km}(120000 \mathrm{~km})$;

2. the observed shock structure at the inbound Halley encounter consisted of a series of velocity dips and recoveries [Coates et al., 1987; Neugebauer et al., 1987a] before the flow settled into its final downstream state;

3. the shock observed on the outbound Halley encounter, although quasi-parallel, possessed an unusually strong rotation of the downstream magnetic field-the "draping shock" of Neubauer et al. [1990](N2).

Clearly, the nature of the cometary shock is poorly understood, although simulations suggest the shock thickness is a consequence of mass-loading upstream of the shock [Omidi \& Winske, 1987]. In particular, Point 1 above distinguishes the cometary shock from ordinary non-reacting gas dynamical or MHD shocks in that mass-loading is important within the shock itself. It was pointed out by N2 and subsequently by Zank \& Oughton [1991] (ZO) that, using typical cometary gas production rates and dissociation lifetimes, the ratio of the newly ionized cometary mass flux injected within the shock to the convected incident mass flux can easily achieve values of $\sim 0.01$. N2 suggested that the Rankine-Hugoniot (R-H) relations should include a source term to account for the significant mass-loading within the body of the shock, suggesting further that mass injection at the shock may provide an explanation for the observed "draping shock". However, a strong rotation of the downstream magnetic field leads to a significant reduction in the downstream gas pressure, and this raised questions concerning the admissibility of certain solutions to the R-H relations [ $Z O$ ]. Simple thermodynamical arguments used in MHD are inappropriate for complex reacting flows [Zank, 1991], and a more sophisticated formulation of the "entropy condition" is required.

In this Letter the approach of $\mathrm{ZO}$ is applied to the case of parallel MHD shocks subjected to mass-loading. We provide a complete characterization of mass-loading parallel MHD shocks and draw attention to four remarkable properties that distinguish such shocks from their classical MHD counterparts. We show that (1) mass-loading introduces shearing stresses into the shock, thereby significantly reducing the parameter regime admitting stable transitions; (2) a tangential magnetic field component is always "switchedon" downstream of the parallel shock; (3) a new class of compound shocks, consisting of a velocity dip followed by a recovery to a supersonic although decelerated downstream state, exists; and (4) the equivalent of parallel slow mode MHD shocks cannot exist in reacting mass-loading media. We stress that we are not considering the shock structure problem. 


\section{Equations and Analysis}

Two important points for our analysis emerge from the observations presented by N2: 1) the thickness of even the shock observed on the outbound Halley encounter is relatively thin when compared to typical gross length scales in the system, thereby validating a 1-D model; 2) the coplanarity theorem is valid for the cometary shock. Thus either the transverse newly ionized gas velocity $V_{t}$ is in the plane spanned by the upstream and downstream magnetic field vectors $B$ or $V_{t}$ is negligibly small. These observations underlie our assumptions.

To include the injection of cometary ions within the shock, one needs an "averaged" source term for the mass in the $\mathrm{R}-\mathrm{H}$ conditions. Conservation of mass then becomes

$$
\left[\rho u_{\varpi}\right] \equiv \rho u_{c}-\rho_{0} u_{x 0}=\alpha \equiv q m_{c} d,
$$

where $q \equiv$ average volume production rate of cometary ions, $m_{c} \equiv$ cometary ion mass, $d \equiv$ observed shock thickness, $\rho$ the fluid density and $u_{x 0}$ the normal upstream fluid velocity. We assume that the pick-up ion velocity distribution is shell-like due to rapid pitch-angle scatteringthis is supported observationally [Neugebauer et al., 1987b] and ensures that the plasma flow is essentially hydrodynamic. Since the newly ionized particles have a very small initial velocity, they add negligibly to the normal momentum balance-however, because mass addition destroys the tangential invariance of the fluid equations, it is important to keep track of the tangential momentum and energy contributions from cometary ions (see below). The remaining R-H conditions are then given by

$$
\begin{aligned}
{\left[\rho u_{x}^{2}+p+B_{y}^{2} / 2 \mu\right] } & =0 ; \\
{\left[\rho u_{x} u_{y}-B_{x} B_{y} / \mu\right] } & =\alpha V_{y} ; \\
{\left[\rho u_{\varepsilon}\left(\varepsilon+1 / 2 u^{2}\right)+u_{x} p+1 / \mu(\mathbf{E} \times \mathbf{B})_{x}\right] } & =\alpha V_{y}^{2} / 2 ; \\
{\left[B_{x}\right]=0 ; \quad\left[u_{x} B_{y}-u_{y} B_{x}\right] } & =0
\end{aligned}
$$

where $\varepsilon$ denotes the internal energy of the fluid, $p$ the pressure, $\mathbf{u}$ the fluid velocity and $\mathbf{E}$ the electric field. A useful simplification is to work in the normal incidence frame of the flow $\bar{u}_{y}=u_{y}-u_{y 0}, \bar{V}_{y}=V_{y}-u_{y 0}$, so that $\bar{u}_{y 0}=0$ and $\bar{V}_{y}$ can be positive, negative or zero. Equations (1)-(5) are effectively unchanged except we now use $\bar{u}_{y}$ and $\bar{V}_{y}$ instead. We omit the "-" hereafter. It is important to recognize that the additional parameter $V_{y}$ can be significant because of the translation and should not therefore be neglected. This was a point emphasized by N2, although they chose to work in the de Hoffmann-Teller frame. The importance of the $V_{y}$ term is a-consequence of the R-H conditions no longer being invariant with respect to tangential flows, and as noted by Coates et al. [1990], $V_{y}$ can be sizeable.

For an ideal gas, and a parallel shock (i.e., $B_{y 0}=0$ ), equations (1)-(5) can be combined as a generalized form of the MHD Hugoniot relating the gas pressure $p$ to the specific volume $\tau=1 / \rho$,

$$
\begin{aligned}
\frac{p}{p_{0}} & =\frac{1-\Gamma \bar{\tau}}{\bar{\tau}-\Gamma}-\frac{\Gamma}{\beta_{p 0}}\left(\frac{\bar{\alpha}}{1+\bar{\alpha}}\right)^{2} \frac{\bar{\tau}-1}{(\bar{\tau}-\Gamma)} \frac{\tilde{V}_{y}^{2}}{\left(\bar{\tau}-\bar{\tau}_{*}\right)^{2}} \\
& +\frac{2 \bar{\alpha}}{1+\bar{\alpha}} \frac{\Gamma}{\beta_{p 0}} \frac{1 / \bar{\tau}_{*}}{(\bar{\tau}-\Gamma)}\left[\tilde{\tau}+\frac{1}{1+\bar{\alpha}} \frac{\tilde{\tau}-(1+\bar{\alpha}) \tilde{\tau}_{k}}{\tilde{\tau}-\tilde{V}_{*}} \bar{V}_{y}^{2}\right]
\end{aligned}
$$

where $\bar{\alpha}=\alpha / m, m=\rho_{0} u_{x 0}, \bar{\tau}=(1+\bar{\alpha}) \tau / \tau_{0}, \Gamma=(\gamma-$ 1) $/(\gamma+1), \gamma$ the adiabatic index of the background fluid and $\bar{V}_{y}=V_{y} / u_{x 00}$. Also, $\beta_{p 0}$ denotes the upstream plasma beta and $\tilde{\tau}_{*}=(1+\bar{\alpha})^{-1} M_{A 0}^{-2}, M_{A 0} \equiv$ the upstream Alfvénic Mach number. With the exception of the term in square brackets, (6) is very similar to the Hugoniot appropriate to oblique non-reacting MHD shocks [Cabannes, 1970], and this is a consequence of mass-loading shearing the flow and dragging the field within the shock. This is evident from the relation describing the downstream tangential magnetic field,

$$
\frac{B_{y}}{B_{x}}=\frac{\bar{\alpha}}{1+\bar{\alpha}} \frac{\tilde{V}_{y}}{\bar{\tau}-\bar{\tau}_{*}}, \quad \tilde{V}_{y}=\frac{V_{y}}{u_{x 0}},
$$

illustrating that mass-loading always "switches on" a shock.

It can be verified that those $\mathrm{R}-\mathrm{H}$ solutions contained within the following regions of the $(\bar{\tau}, p)$-plane

$\bar{\tau}<(>) \frac{1}{1+\bar{\alpha}}$ and $\frac{p}{p_{0}}>(<) 1-\frac{1}{\beta_{p 0}}\left(\frac{\bar{\alpha}}{1+\bar{\alpha}}\right)^{2} \frac{\bar{V}_{y}^{2}}{\left(\bar{\tau}-\bar{\tau}_{m}\right)^{2}}$

do not satisfy $m^{2}>0$. Solutions to the $R-H$ conditions occur at the intersection of the Hugoniat (6) and the generalized Rayleigh curve

$$
\frac{p}{p_{0}}=\left(1+\frac{2}{\beta_{p 0}} \frac{1}{1+\bar{\alpha}} \frac{1}{\bar{\tau}_{*}}\right)-\frac{1}{\beta_{p 0}}\left(\frac{\bar{\alpha}}{1+\bar{\alpha}}\right)^{2} \frac{\tilde{V}_{\nu}^{2}}{\left(\tilde{\tau}-\bar{\tau}_{*}\right)^{2}}-\frac{2}{\beta_{p 0}} \frac{\tilde{\tau}}{\bar{\tau}_{k}}
$$

Unlike classical MHD, (6) and (9) need not intersect, indicating that a state cannot be connected to itself by a massloading front. However, distinguished points, analogous to the Chapman-Jouguet points of combustion theory, do exist at which the Hugoniot and Rayleigh curves touch. At these points of common tangency the downstream normal fluid velocity $u_{x}$ must satisfy

$$
u_{\infty}^{4}-\left(\gamma p / \rho+B^{2} / \mu \rho\right) u_{\infty}^{2}+(\gamma p / \rho)\left(B_{\approx}^{2} / \mu \rho\right)=0,
$$

which is equivalent to the magnetosonic dispersion relation. Thus, at the "Chapman-Jouguet" points, the downstream fluid velocity $u_{x}$ is equal to either the fast or slow magnetosonic speed. Relations (8) and (10) enable one to distinguish between sub-magnetosonic and sub-Alfvénic downstream flow speeds.

Two examples of the Hugoniot for $\beta_{p 0}=1$ are illustrated in Figure 1. I and II identify the mass-loading "Chapman-Jouguet" points and the inadmissible section of the Hugoniot, MN, lies in the unshaded region. Increasing the upstream plasma beta to 5 now introduces three distinct tangency points I, II, III (Figure 2), each corre-

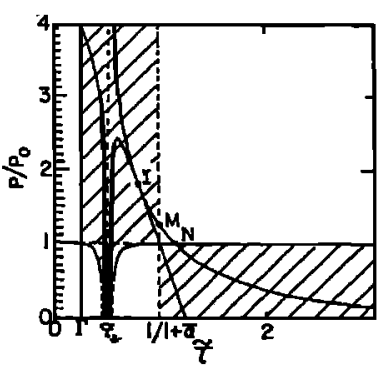

(a)

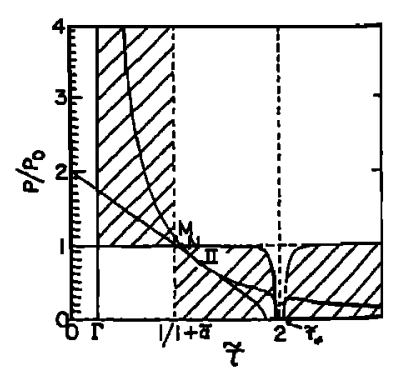

(b)
Fig. 1: Hugoniots for $\beta_{p 0}=1, V_{y}=4, \gamma=5 / 3, \bar{\alpha}=0.01$. 


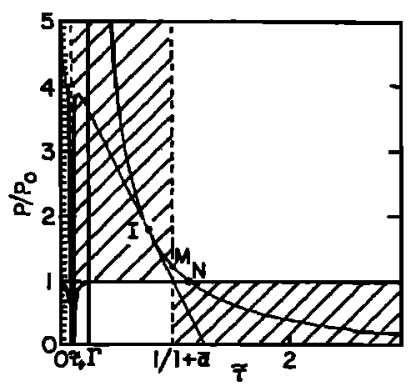

(a)



(b)

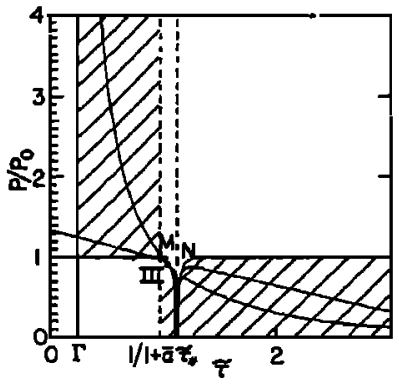

(c)

Fig. 2: Hugoniots for $\beta_{p 0}=5, V_{y}=4, \gamma=5 / 3, \bar{\alpha}=0.01$.

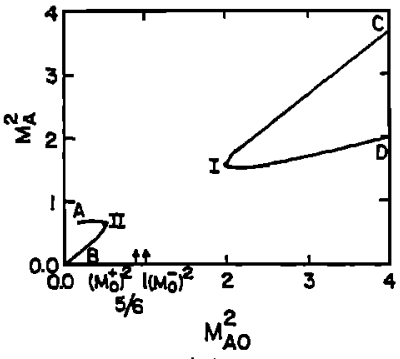

(a)

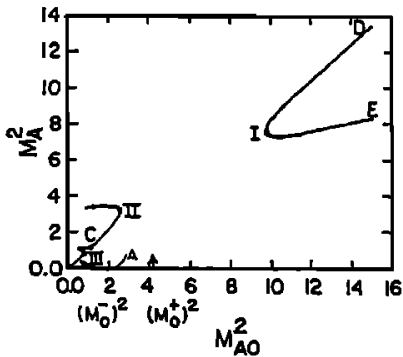

(b)
Fig. 3: Shock polar relations appropriate to (a) Fig. 1, (b) Fig. 2. $\left(M_{0}^{-}\right)^{2}=V_{s 0}^{2} / V_{A 0}^{2}=1,\left(M_{0}^{+}\right)^{2}=V_{f 0}^{2} / V_{A 0}^{2}=\gamma \beta_{p 0} / 2$.

sponding to a different upstream state. This is illustrated in mass-loading MHD shock polar plots (Figure 3) relating the square of the upstream and downstream Alfvénic Mach numbers. Points I, II and III of figure 3 correspond to the "Chapman-Jouguet" points of the Hugoniot. It is noticeable, on comparing Figure 3 to examples of non-reacting MHD shock polar plots [e.g., Webb et al., 1987], that large regions of upstream parameter space no longer admit real solutions to the R-H relations.

To determine which of the marked sections on the massloading shock polar plots correspond to physically admissible transitions, we use a formulation of the entropy condition described in detail by $\mathrm{ZO}$. The characteristics for our reacting system are the same as those for classical MHD i.e., $C_{s, f, A}^{ \pm}: \mathrm{d} x / \mathrm{d} t=u_{a} \pm V_{s, f, A}, V_{s, f, A}$ the slow, fast magnetosonic speed or the Alfvén speed. For the $\beta_{p 0}=1$ case, consider first an upstream state $S$ with $p>p_{I}$, for which $u_{x 0}>V_{f 0}$ (Figure's 1,3). In this case, the downstream flow velocity satisfies $V_{A}<u_{m}<V_{f}$. The characteristics for a stationary shock are illustrated in Figure $4 \mathrm{a}$ for this case, showing that the shock separates only the $C_{f}^{-}$charac- teristic. Since the $C_{f}^{-}$characteristics on either side of the shock can be traced back to the initial data, this massloading front satisfies the geometrical entropy condition and therefore represents a physically admissible solution to the $\mathrm{R}-\mathrm{H}$ conditions. The $C_{f}^{-}$front is the mass-loading analogue of the fast mode MHD shock. Similarly, for the case $p=p_{I}$ (i.e., $S=I$ ), we have $u_{x 0}>V_{f 0}$ and $u_{x}=V_{f}$ downstream. Here the downstream characteristic coincides with the mass-loading front (Figure $4 \mathrm{~b}$ ), but this is still sufficient to allow us to determine the downstream state without further assumption. The weak compression case, for which $p_{I}>p>p_{M}>p_{0}$, has $u_{x 0}>V_{f 0}$ ahead of the front and $u_{x}>V_{f}$ behind. Nevertheless, the flow is decelerated since $u_{\varepsilon}<u_{x 0}$ (Figure's 1,3). In this case, the characteristics are not separated (hence the mass-loading system is non-convex) (Figure 4c) and to exclude discontinuities that move faster than the characteristics on both sides, it is necessary to use a compound wave to connect upstream and downstream states [ZO]. This is accomplished by connecting the upstream state to the $S=I$ front and then following by an isentropic centered rarefaction wave to reach the downstream state. Such a compound wave is possible because the $S=I$ front moves at the downstream fast magnetosonic speed. If we use this compound wave to connect the states, then the downstream state is determined completely by the upstream state. Had we not excluded weak compression discontinuities, then the solution to the initial value problem would not be unique. The weak and strong expansion states (figures 4d and e) are seen to be indeterminate and inadmissible solutions to the R-H conditions respectively. Consequently, we may identify the segment ID of Figure 3a with fast mode mass-loading shocks, IC with a new form of MHD shock, the compound shocks, AII as inadmissible, and BII with subsonic-subsonic expansion flows which may or may not be admissible.

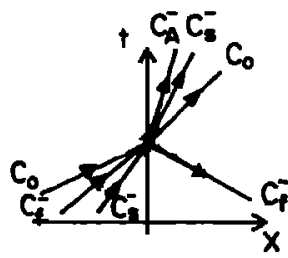

(a)

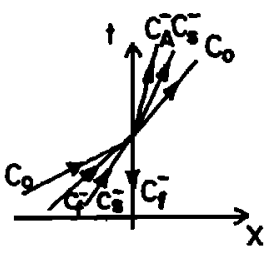

(b)

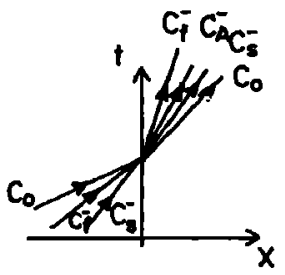

(c)

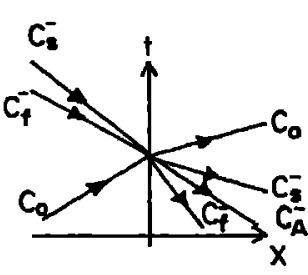

(d)



(e)

Fig. 4: Characteristics appropriate to possible solutions of the R-H conditions for $\beta_{p 0}=1$ (Fig. 1). 


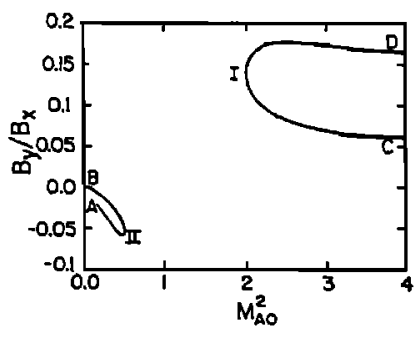

(a)

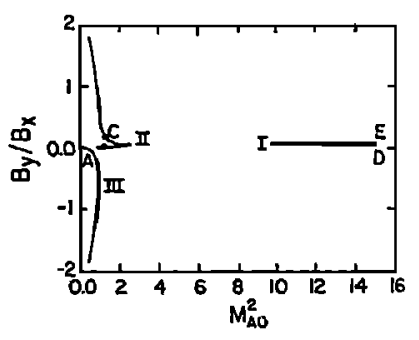

(b)
Fig. 5: Downstream tangential magnetic fields for (a) Fig. 1, (b) Fig. 2.

An equivalent analysis holds for the $\beta_{p 0}=5$ case, with IE and ID corresponding to fast mode mass-loading and compound shocks respectively. The remaining R-H solutions are inadmissible. Thus, from the above analysis, classical parallel slow mode MHD shocks have no mass-loading counterpart. Finally the "switched on" tangential magnetic field is plotted in Figure 5. Clearly, because $M_{A 0}$ can never approach 1 , extremely strong rotations of the magnetic field cannot occur, although modest rotations of up to $10^{\circ}$ are possible. This is probably insufficient to account properly for the magnetic field observations described by N2, and one needs to consider the full shock structure problem.

\section{Conclusions}

Observational evidence obtained on the missions to GZ and Halley suggests that non-reacting MHD may be an inappropriate description for cometary shocks. The observed thickness of the shock structures implies that mass-loading must be significant within the shock, and, as such, suggests that mass-loading shocks have more in common with combustion theory than with gas dynamics. The results presented here have important implications, both for observations as well as numerical modelling. Firstly, in investigating the observed properties of cometary shocks, for example, simple gas dynamical or MHD R-H conditions are assumed-however, as we've discussed, these results should be interpreted with some caution. Secondly, considerable insight into the interaction of the solar wind with comets has been gained through numerical simulations. However, many standard numerical schemes use a Riemann solver in the code and if the Riemann solver is appropriate to non-reacting gas dynamics or MHD, the code may well be solving the Riemann problem incorrectly!

In conclusion, we reiterate the properties of parallel massloading shocks: (1) stable parallel mass-loading shocks do not exist for a large parameter regime of upstream states, unlike classical MHD; (2) shearing stresses always rotate the downstream magnetic field; (3) a new class of MHD shocks, viz., compound shocks, has been described, and it is possible that such structures were observed on the inbound Halley encounter, and (4) stable parallel slow mode mass-loading fronts do not excist.

Acknowledgements. The work of GPZ and SO has been supported in part by the Bartol NASA SPT Program and GMW by NASA grant NSG-7101 and NSF grant ATM-
8317701. FMN thanks N.F. Ness for his hospitality and we thank him for useful discussions.

\section{References}

Axford, W.I., The interaction of solar wind with comets, Planet. Space Sci., 12, 719 (1964).

Biermann, L., B. Brosowski \& H.U. Schmidt, The interaction of the solar wind with a comet, Solar Phys., 1, 254 (1967).

Cabannes H., Theoretical Magnetofluiddynamics, Academic Press, New York (1970).

Coates, A. et al., Giotto measurements of cometary and solar wind plasma at the comet Halley bow shock, Nature, 327, 489 (1987).

Coates, A. et al., Plasma parameters near the comet Halley bow shock, J. Geophys. Res., 95, 20701 (1990).

Ip, W.-H. \& W.I. Axford, The Plasma, in Physics and Chemistry of Comets, ed. W.F. Huebner, SpringerVerlag, Berlin-Heidelberg, 177 (1989).

Neubauer, F.M. et al., First results from the Giotto magnetometer experiment at comet Halley, Nature, 321, 352 (1986).

Neubauer, F.M. et al., Giotto magnetic field observations at the outbound quasi-parallel bow shock of comet Halley, Ann. Geophys., 8, 463 (1990).

Neugebauer, M. et al., The variation of protons, alpha particles and the magnetic field across the bow shock of comet Halley, Geophys. Res. Lett., 14, 995 (1987a).

Neugebauer, M. et al., The pick-up of cometary protons by the solar wind; Astron. Astrophys., 187, 21 (1987b).

Omidi, N. and D. Winske, A kinetic study of solar wind mass loading and cometary bow shocks, J. Geophys. Res., 92, 13409 (1987).

Ogino, T., R.J. Walker \& M. Ashour-Abdalla, A threedimensional MHD simulation of the interaction of the solar wind with comet Halley, J. Geophys. Res., 93, 9568 (1988)

Wallis, M.K., Shock-free deceleration of the solar wind?, Nature, 233, 23 (1971).

Webb, G.M., G.P. Zank \& J.F. McKenzie, Relativistic oblique mhd shocks, J. Plasma Phys., 37, 117 (1987).

Zank, G.P., Weyl's theorem for MHD, J. Plasma Phys., 46, 11 (1991).

Zank, G.P. \& S. Oughton, Properties of mass-loading shocks: I. Hydrodynamic considerations, J. Geophys. Res., 96, 9439 (1991).

G.P. Zank and S. Oughton, Bartol Research Institute, The University of Delaware, Newark, DE 19716, USA.

F.M. Neubauer, Institut für Geophysik und Meteorologie, Universität Köln, Albertus-Magnus-Platz, $5000 \mathrm{Köln-}$ 41, F.R.G.

G.M. Webb, Department of Planetary Sciences, University of Arizona, Tucson, AZ 85721, USA.

(Recieved: May 3, 1991

Accepted: August 19, 1991) 